\title{
THE RYJÁČEK CLOSURE AND A FORBIDDEN SUBGRAPH
}

\author{
AKIRA SAITO \\ Department of Information Science \\ Nihon University \\ Sakurajosui 3-25-40 \\ Setagaya-Ku, Tokyo 156-8550, Japan \\ e-mail: asaito@chs.nihon-u.ac.jp \\ AND \\ LIMING XIONG \\ School of Mathematics and Statistics \\ Beijing Institute of Technology \\ Beijing 100081, China \\ and \\ Beijing Key Laboratory on MCAACI \\ Beijing Institute of Technology \\ Beijing 100081, China \\ e-mail: lmxiong@bit.edu.cn
}

\begin{abstract}
The Ryjáček closure is a powerful tool in the study of Hamiltonian properties of claw-free graphs. Because of its usefulness, we may hope to use it in the classes of graphs defined by another forbidden subgraph. In this note, we give a negative answer to this hope, and show that the claw is the only forbidden subgraph that produces non-trivial results on Hamiltonicity by the use of the Ryjáček closure.
\end{abstract}

Keywords: closure, claw-free graph, Hamiltonian graph, perfect matching, traceable graph.

2010 Mathematics Subject Classification: 05C45, 05C70.

\section{REFERENCES}

[1] G. Chartrand, L. Lesniak and P. Zhang, Graphs \& Digraphs (5th Ed.) (Chapman and Hall/CRC, Boca Raton, Florida, USA, 2010). 
[2] M. Jünger, W.R. Pulleyblank and G. Reinelt, On partitioning the edges of graphs into connected subgraphs, J. Graph Theory 9 (1985) 539-549. doi:10.1002/jgt.3190090416

[3] M. Las Vergnas, A note on matchings in graphs, Colloque sur la Théorie des Graphes, Cahiers Centre Études Rech. Opér. 17 (1975) 257-260.

[4] M.M. Matthews and D.P. Sumner, Hamiltonian results in $K_{1,3}$-free graphs, J. Graph Theory 8 (1984) 139-146. doi:10.1002/jgt.3190080116

[5] D.J. Oberly and D.P. Sumner, Every connected, locally connected nontrivial graph with no induced claw is Hamiltonian, J. Graph Theory 3 (1979) 351-356. doi:10.1002/jgt.3190030405

[6] M.D. Plummer and A. Saito, Forbidden subgraphs and bounds on the size of a maximum matching, J. Graph Theory 50 (2005) 1-12. doi:10.1002/jgt.20087

[7] Z. Ryjáček, On a closure concept in claw-free graphs, J. Combin. Theory Ser. B 70 (1997) 217-224. doi:10.1006/jctb.1996.1732

[8] D.P. Sumner, 1-factors and antifactor sets, J. Lond. Math. Soc. (2) 13 (1976) 351-359. doi:10.1112/jlms/s2-13.2.351

[9] C. Thomassen, Reflections on graph theory, J. Graph Theory 10 (1986) 309-324. doi:10.1002/jgt.3190100308

Received 29 May 2015

Revised 30 September 2015

Accepted 30 September 2015 